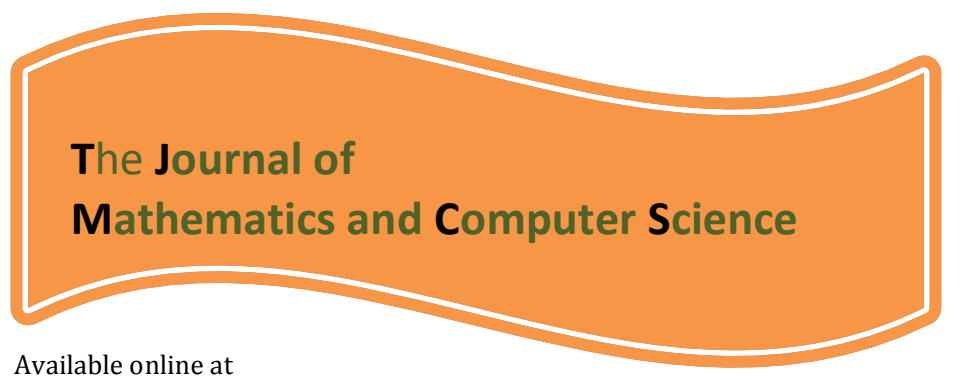

http://www.TJMCS.com

The Journal of Mathematics and Computer Science Vol. 4 No.1 (2012) 71 - 80

\title{
Adaptive robust PID controller design based on a sliding mode for uncertain chaotic systems
}

\author{
Yaghoub heidari ${ }^{1}{ }^{*}$, Rashin nimaee rad $^{2}$ \\ 1Department of Electrical, Nour Branch, Islamic Azad University, Nour, Iran \\ 2Department of Mathematics, Nour Branch, Islamic Azad University, Nour, Iran
}

Received: November 2011, Revised: February 2012

Online Publication: May 2012

\begin{abstract}
A robust adaptive PID controller design motivated from the sliding mode control is proposed for a class of uncertain chaotic systems in this paper. Three PID control gains, $\mathrm{K}_{\mathrm{p}}, \mathrm{K}_{\mathrm{i}}$, and $K_{d}$, are adjustable parameters and will be updated online with an adequate adaptation mechanism to minimize a previously designed sliding condition. By introducing a supervisory controller, the stability of the closed-loop PID control system under with the plant uncertainty and external disturbance can be guaranteed. Finally, a well-known Vanderpol oscillator is used as an illustrative to show the efectiveness of the proposed robust a PID controller.
\end{abstract}

Keywords: Robust, PID, Adaptive, Vanderpol, Chaos

\section{Introduction}

The presence of chaos in physical systems has been extensively demonstrated. Chaotic systems are rather complex dynamical nonlinear system and the classical features appeared in output responses of chaotic systems have, for example, excessive sensitivity to initial conditions, broad spectrums of Fourier transform, and fractal properties of the motion in the phase space [1-3]. Due to its powerful applications in chemical reactions, power converters, and information

\footnotetext{
1,* Corresponding Author: Yaghoub heidari, Department of Electrical, Nour Branch, Islamic Azad University, Nour, Iran

E-mail address: masudheidari@yahoo.com (Phone: +98 122625 4590)
} 
processing, etc., it is meaningful and practical to design certain control law to cope with these complex chaotic systems for real engineering applications. To do this, different techniques have been developed to achieve the chaotic control. For example, sliding mode control [1-3], bangbang control [4], optimal control [5,6], intelligent control base on using neural networks [7], feedback linearization [8], differential geometric method [9], adaptive control [10-12], and among many others [13].

On the other hand, the use of PID control has a long history in control engineering and is acceptable for many real applications due to its simplicity in architecture. Hence, in many real industrial applications, the PID controller is still widely used even though lots of new control techniques have been proposed. However, to our knowledge, the control of chaotic system by using simple PID control has not been well discussed.

The key for designing a PID controller is the determination of three parameters of PID controller, i.e., proportional gain $K_{p}$, integral gain $K_{i}$, and derivative gain $K_{d}$. In the past, the PID self-tuning methods based on the relay feedback technique have often presented for a class of SISO systems [14-17] and MIMO systems [18]. In general, the controlled plants considered in the above-proposed PID control systems mostly belong to linear systems plus time delays. Moreover, in recent years, a new and attractive control strategy that is to combine neural networks and/or fuzzy systems with the traditional adaptive control has been proposed for nonlinear systems. Two types of adaptive controls are generally investigated:

Indirect adaptive control. The unknown model of nonlinear system is first obtained and learned by using the neural/ fuzzy system and then a feedback linearization control law is designed based on this model [19-22].

Direct adaptive control. The neural/fuzzy system is directly used as a controller in the feedback control system, i.e., the output of neural/fuzzy system is a control input to the nonlinear plant. Usually, an error signal between the desired and actual outputs is employed to online update adjustable parameters in the neural/fuzzy controller [21-25].

In this paper, based on the use of the sliding mode, a robust adaptive PID control tuning is newly proposed to cope with the control problem for a class of uncertain chaotic systems with external disturbance. Three gains of PID controller, i.e., $\mathrm{K}_{\mathrm{p}}$, Ki, and $\mathrm{K}_{\mathrm{d}}$, are regarded as adjustable parameters and will be adjusted during control procedure. We wish to update these parameters with a proper adaptation mechanism such that a previously designed sliding condition is minimized as possibly. The stability of closed-loop PID control system can be guaranteed by using the Lyapunov approach with a supervisory controller [19, 21, 22, 26 ]. The detailed descriptions for the PID control gains tuning and for the analysis of system stability will be presented in the following.

\section{PID CONTROLLER}

The continuous form of a PID controller, with input $e(t)$ and output $u_{p i d}(t)$, is generally given as

$$
u_{p i d}=K_{p} e(t)+K_{i} \int_{0}^{t} e(\tau) d \tau+K_{d} \frac{\mathrm{d}}{\mathrm{d} t} e(t)
$$

Where, $K_{p}$ is the proportional gain, $T_{i}$ is the integral time constant, and $T_{d}$ is the derivative time constant, where $K_{i}=K_{p} / T_{i}$ is the integral gain and $K_{d}=K_{p} T_{d}$ is the derivative gain

\section{3. . System definition for uncertain chaotic systems}

For simplification, we only consider a second-order uncertain chaotic systems, but the approach 
can be generalized to high-order systems. With the input $u(t) \in \mathfrak{R}$ and the output $y(t) \in \mathfrak{R}$, the uncertain chaotic system is described as

$$
\begin{aligned}
& \dot{x}_{1}=x_{2} \\
& \dot{x}_{2}=-\omega x_{1}+\Delta f(X, t)+u(t) \\
& y(t)=x_{1}(t)
\end{aligned}
$$

Where, $X=\left[\begin{array}{ll}x_{1} & x_{2}\end{array}\right]^{T}$ are measurable states vector of the system, $\Delta f($.$) is the plant uncertainty$ applied to the system . Let $E=Y_{d}-X=\left[\begin{array}{ll}e & \dot{e}\end{array}\right]^{T}$ be an error signal between the desired and actual outputs. Define $Y_{d}=\left[\begin{array}{ll}y_{d} & \dot{y}_{d}\end{array}\right]^{T}$ and assume that both $y_{d}$ and $\dot{y}_{d}$ are bounded, i.e., $|\Delta f().| \leq \Delta f_{u}($.$) .$ Then the error vector of system becomes $E=Y_{d}-X=\left[\begin{array}{ll}e & \dot{e}\end{array}\right]^{T}$. Suppose that we can choose a gain vector $K=\left[\begin{array}{ll}k_{0} & k_{1}\end{array}\right]^{T}$ such that roots of $s^{2}+k_{1} s+k_{0}=0$ are in the open left-half complex plane. Now let a feedback linearization controller be given by

$$
u_{F . L .}=\omega x_{1}-\Delta f(X, t)+\ddot{y}_{d}+K^{T} E
$$

Substituting (3) into (2), we have

$$
\ddot{e}+k_{1} e+k_{0} e=0
$$

Consequently, from (5), we have e $\lim _{t \rightarrow \infty} e(t) \rightarrow 0$ i.e., $y=y_{d}$ asymptotically.

Assumption 1. Let the constraint set $\Omega_{x}$ for the state $X$ can be defined as

$$
\Omega_{x}=\left\{X \in \mathfrak{R}^{2}:\|X\| \leq M_{x}\right\}
$$

Where $M_{x}$ is a pre-specified parameter

It is desired that the state trajectory of system $X$ never reach the boundary of $\Omega_{x}$ during the control procedure. For simplicity of analysis, we may choose $M_{x} \leq\left\|Y_{d}\right\|_{\infty}$.

\section{Supervisory controller design and adaptation laws for PID controller} Now let the control input in (2) be given by

$$
u=u_{p i d}+u_{s}
$$

where $u_{\text {pid }}$ is the PID controller as shown in (2) and $u_{s}$ is the extra supervisory controller that will be fired only when the states of the system exceeds some bound and guarantees the stability of the system. Based on concepts of sliding mode control, a proper adaptation law that is based on the use of gradient method is presented to minimize a designed sliding condition for updating PID control gains

\subsection{Supervisory controller design}

To design the supervisory controller us, substituting (6) into (2), we have, in view of (3), 


$$
\begin{aligned}
\dot{x}_{2} & =-\omega x_{1}+\Delta f(X, t)+u_{p i d}+u_{s} \\
& =-\omega x_{1}+\Delta f(X, t)+u_{p i d}+u_{s}+u_{F . L .}-u_{F . L .} \\
& =\ddot{y}_{d}+K^{T} E-\left[-u_{p i d}-u_{s}+u_{F . L .}\right]
\end{aligned}
$$

This implies that

$$
\ddot{e}=-K^{T} E+\left[-u_{p i d}-u_{s}+u_{F . L .}\right]
$$

Let

$$
\begin{aligned}
& A=\left[\begin{array}{rr}
0 & 1 \\
-k_{0} & -k_{1}
\end{array}\right], \\
& B=\left[\begin{array}{ll}
0 & 1
\end{array}\right]^{T}
\end{aligned}
$$

be a companion form. From (8) and (9), we have

$$
\dot{E}=A E+B\left[u_{F . L .}-u_{p i d}-u_{s}\right]
$$

Now consider a Lyapunov function candidate

$$
V=\frac{1}{2} E^{T} P E
$$

Where, $p$ is a positive definite symmetric matrix satisfying the Lyapunov equation

$$
A^{T} P+P A=-Q
$$

And $Q$ is a positive definite symmetric matrix selected by designer. Define

$$
V_{m}=\frac{1}{2} \lambda_{m}\left(M_{x}-\left\|Y_{d}\right\|_{\infty}\right)^{2}
$$

Where $\lambda_{m}$ denotes the minimum eigenvalue of $p$. Note that if $\|X\| \geq M_{x}$, then, from (10), we have

$$
\begin{aligned}
V & \geq \frac{1}{2} \lambda_{m}\|E\|^{2} \geq \frac{1}{2} \lambda_{m}\left(\|X\|-\left\|Y_{d}\right\|\right)^{2} \\
& \geq \frac{1}{2} \lambda_{m}\left(M_{x}-\left\|Y_{d}\right\|_{\infty}\right)^{2}=V_{m}
\end{aligned}
$$

Hence if $V<V_{m}$, then $\|X\|<M_{x}$. Moreover, the time derivative of $V$ along the trajectories of the closed-loop system (10) satisfies

$$
\begin{aligned}
\dot{V} & =\frac{1}{2} E^{T}\left(A^{T} P+P A\right) E+E^{T} P B\left[u_{F . L .}-u_{p i d}-u_{s}\right] \\
& =-\frac{1}{2} E^{T} Q E+E^{T} P B\left[u_{F . L .}-u_{p i d}-u_{s}\right] \\
& \leq-\frac{1}{2} E^{T} Q E+\left|E^{T} P B\right|\left(\left|u_{F . L .}\right|+\left|u_{p i d}\right|\right)-E^{T} P B u_{s}
\end{aligned}
$$

From (4) and hypotheses of $|\Delta f().| \leq \Delta f_{u}$ (.) we have

$$
u_{F . L .} \leq \omega\left|x_{1}\right|+\Delta f_{u}(.)+\left|\ddot{y}_{d}\right|+\left|K^{T} E\right|
$$

Hence, if the supervisory controller is chosen as

$$
\begin{aligned}
u_{s}= & \operatorname{sgn}\left(E^{T} P B\right)\left[\omega\left|x_{1}\right|+\Delta f_{u}(.)\right. \\
& \left.+\left|\ddot{y}_{d}\right|+\left|K^{T} E\right|+\left|u_{p i d}\right|\right]
\end{aligned}
$$

Then, from (14) and (15), we can guarantee that $\dot{V}<0$ in (13) if $V \geq V_{m} \quad[21,22$, and 26]. Chattering 


$$
\begin{aligned}
u_{s}= & \operatorname{sat}\left(E^{T} P B\right)\left[\omega\left|x_{1}\right|+\Delta f_{u}(.)\right. \\
& \left.+\left|\ddot{y}_{d}\right|+\left|K^{T} E\right|+\left|u_{p i d}\right|\right]
\end{aligned}
$$

\subsection{Adaptive laws for PID controller tuning}

On the other hand, in order to derive a proper adaptation law to update three control gains, let us define a designed signal $x_{d}$ as

$$
\dot{x}_{d}=\ddot{y}_{d}+k_{1} \dot{e}+k_{0} e
$$

Moreover, a sliding surface is defined as

$$
S=x_{2}-x_{d}
$$

If the sliding mode occurs, i.e., $S=0$, then

$$
S=0
$$

Therefore, the sliding condition is defined as

$$
\dot{V}=S \dot{S}<0
$$

Using, (2) and (20), we have:

$$
\dot{S}=\dot{x}_{2}-\dot{x}_{d}=-\omega x_{1}+\Delta f(X, t)+u(t)-\dot{x}_{d}
$$

Substituting (6) into (20) and multiplying both sides of (20) by S, the following is obtained:

$$
S \dot{S}=S\left[-\omega x_{1}+\Delta f(X, t)+u_{p i d}(t)+u_{s}(t)-\dot{x}_{d}\right]
$$

Based on the gradient method and the chain rule, and using (2) and (21), the adaptation laws for three control gains $K_{p}, K_{i}$, and $K_{d}$ can be easily obtained as follows:

$$
\begin{gathered}
\dot{K}_{p}=-\gamma \frac{\partial S \dot{S}}{\partial K_{p}}=-\gamma \frac{\partial S \dot{S}}{\partial u_{p i d}} \frac{\partial u_{p i d}}{\partial K_{p}}=-\gamma S e(t) \\
\dot{K}_{i}=-\gamma \frac{\partial S \dot{S}}{\partial K_{i}}=-\gamma \frac{\partial S \dot{S}}{\partial u_{p i d}} \frac{\partial u_{p i d}}{\partial K_{i}}=-\gamma S \int_{0}^{t} e(\tau) d \tau \\
\dot{K}_{d}=-\gamma \frac{\partial S \dot{S}}{\partial K_{d}}=-\gamma \frac{\partial S \dot{S}}{\partial u_{p i d}} \frac{\partial u_{p i d}}{\partial K_{d}}=-\gamma S \frac{\mathrm{d}}{\mathrm{d} t} e(t)
\end{gathered}
$$

Where $\gamma$ is the learning rate. Notice that if the learning rate $\gamma$ or the initial values of PID control gains are not selected adequately, the resulted PID controller will probably make the states of system divergent. Fortunately, the supervisory controller as in (15) will play an important role that provides an extra input to pull the states back to the pre-specified constraint set $\Omega_{x}$ and also guarantees the stability of the system. 


\section{Implementation of a adaptive robust PID controller on Vanderpol oscillator}

The plant of interest considered in this study is a Vanderpol oscillator, and its nominal dynamic equations are described as [1]

$$
\begin{aligned}
& \dot{x}_{1}=x_{2} \\
& \dot{x}_{2}=-x_{1}+\varepsilon x_{2}\left(1-x_{1}^{2}\right)+u(t) \\
& y(t)=x_{1}(t)
\end{aligned}
$$

Where, $0<\varepsilon<2$, with comparing (28) to (2) it can be resulted $\Delta f(X, t)=\varepsilon x_{2}\left(1-x_{1}^{2}\right)$ , $\omega=1$

The sampling time is equal to 0.01 and the initial states of the system are assumed to be $x_{1}(0)=0.1$ and $x_{2}(0)=0$. For obtaining $\Delta f_{u}\left(\right.$.) we must have $|\Delta f().| \leq \Delta f_{u}($.)

$$
|\Delta f(.)|=\left|\varepsilon x_{2}\left(1-x_{1}^{2}\right)\right| \leq 2\left|x_{2}\right|\left(1+x_{1}^{2}\right)=\Delta f_{u}(.)
$$

The proposed robust adaptive PID controller is now applied to control the uncertain chaotic system with external disturbance. We wish that the output of uncertain chaotic system $y(t)$ can track the desired output $y_{d}=\sin (t)$. Also we choose $k_{0}=9$ and $k_{1}=6$ so that the roots of $\mathrm{s}^{2}+\mathrm{k} 1 \mathrm{~s}$ $+\mathrm{k} 0=0$ are in the left-half complex plane and let the learning rate $\gamma=1$. From (9), we have

$$
A=\left[\begin{array}{cc}
0 & 1 \\
-9 & -6
\end{array}\right]
$$

From (12) with $Q=\operatorname{diag}[18,18]]$, we have

$$
P=\left[\begin{array}{cc}
45 / 6 & 1 \\
1 & 1 / 6
\end{array}\right]
$$

Moreover, the adaptation laws in (25)-(27) are used to online update the PID controller with initial gains $K_{d}(0)=K_{p}(0)=K_{i}(0)=5$. The value of $M_{x}$ is deliberately selected to be large enough such that the system state $X$ never reach the boundary of $\Omega_{x}$. The results are demonstrated in Figs. 1-9. Fig. 1 and 3 shows the time trajectories of the controller $u(t)$. Figs. 4 and 5, respectively, show the output responses of $x_{1}$ and $x_{2}$ of the controlled vanderpol system. It can be easily seen from these results that the states of such a chaotic system can quickly track the desired states about after $5 \mathrm{~s}$.

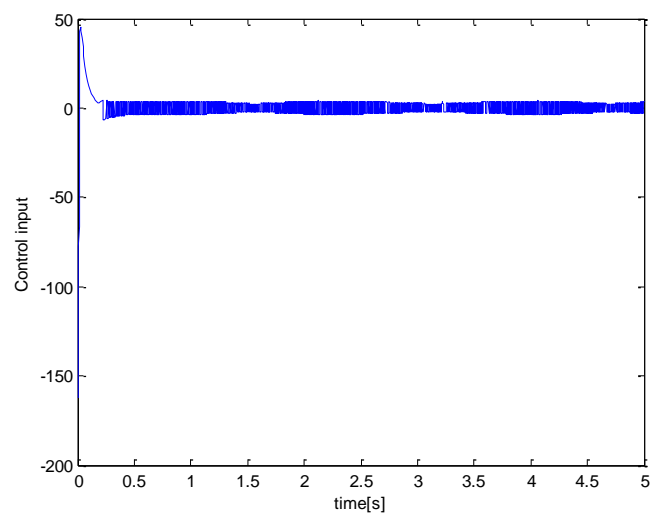

Fig1: control input $\mathrm{U}(\mathrm{t})$ 
Yaghoub heidari, Rashin nimaee rad/ TJMCS Vol. 4 No. 1 (2012) 71 - 80

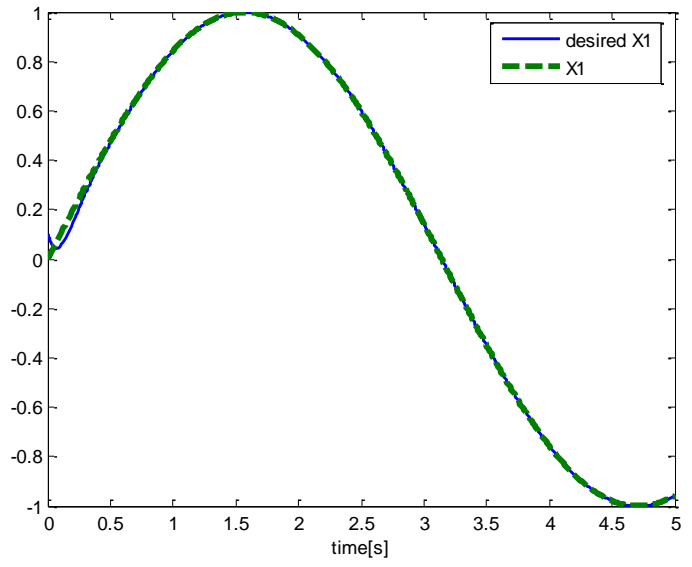

Fig2: Output Result $x_{1}$

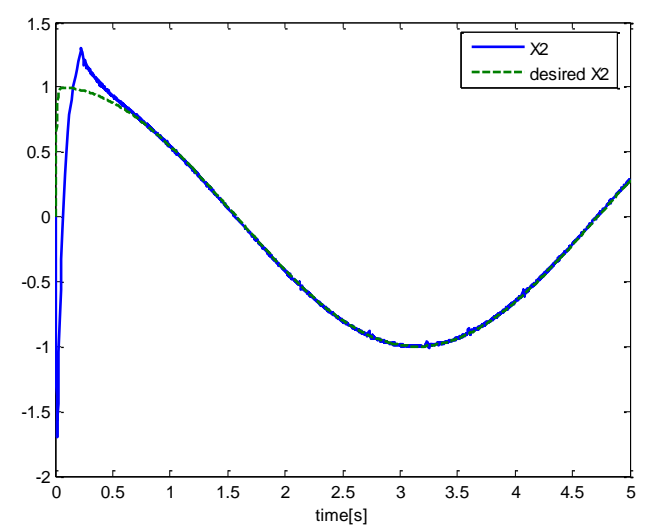

Fig3:Output Result $x_{2}$

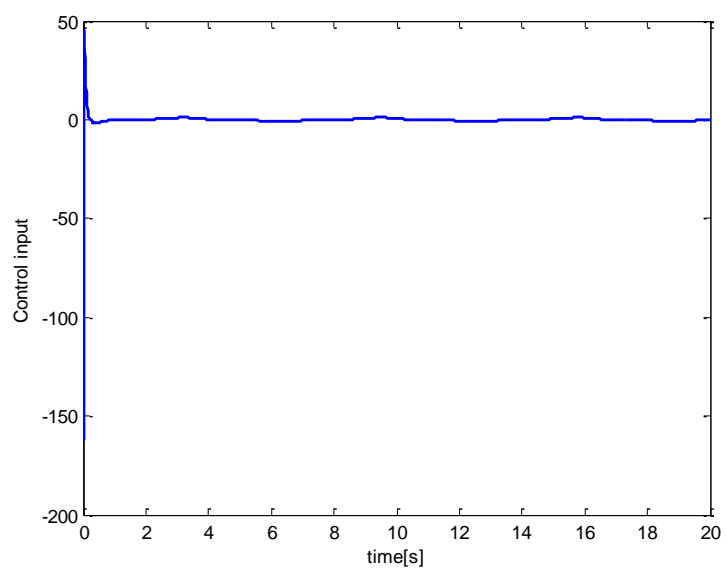

Fig4: control input $U(t)$ 
Yaghoub heidari, Rashin nimaee rad/ TJMCS Vol. 4 No. 1 (2012) 71 - 80

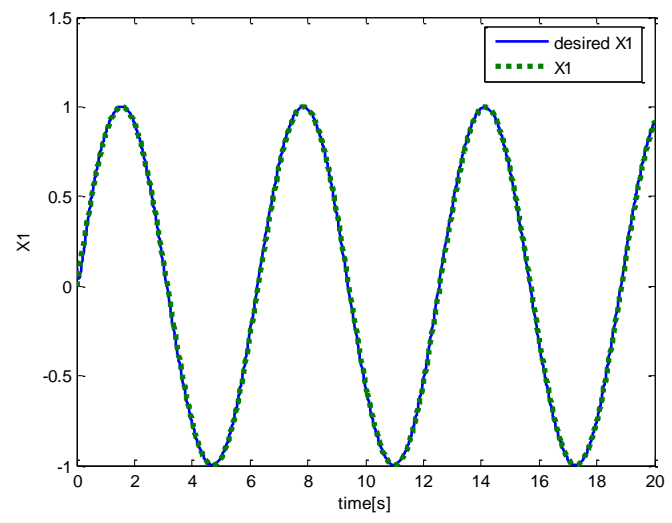

Fig5: Output Result $x_{1}$

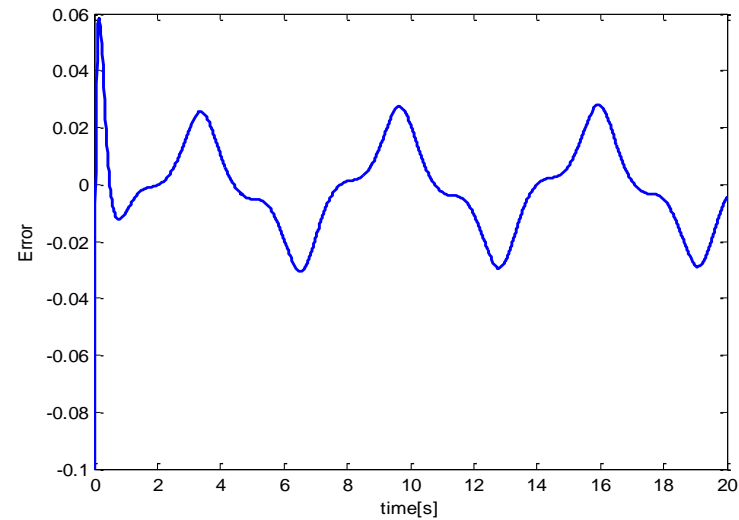

Fig6: Error between output result of $x_{1}$ and $y_{d}$

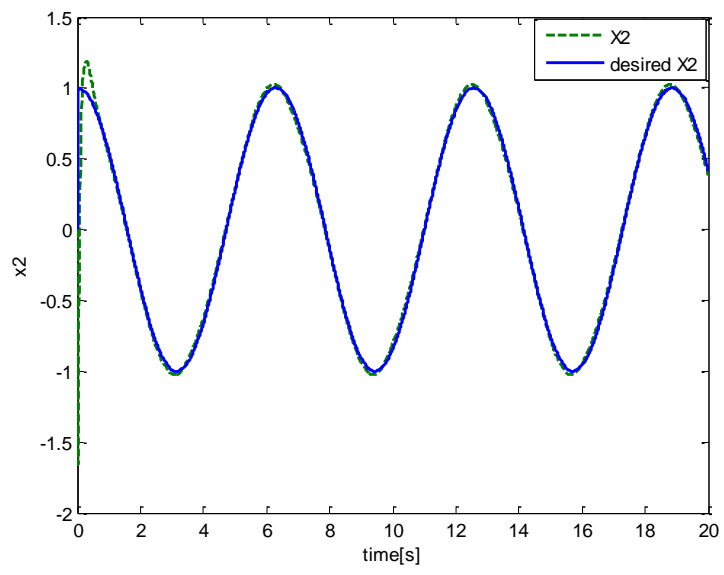

Fig7: Output Result $x_{2}$ 


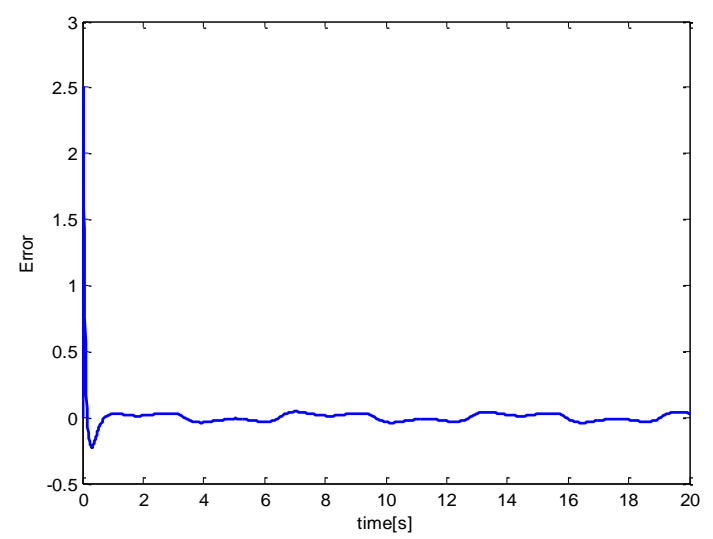

Fig8: Error between output result of $x_{2}$ and $y_{d}$

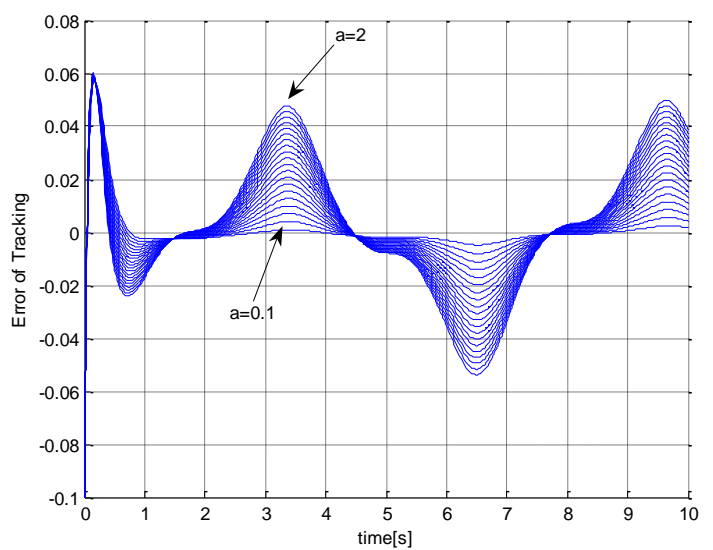

Fig9:Output tracking error respect to variation of

$a$

\section{Conclusions}

In this paper, a robust adaptive PID controller for a class of uncertain chaotic systems has been proposed. The proposed adaptation law for PID control gains tuning is to minimize the designed sliding condition that is motivated from the sliding mode control. Moreover, based on the use of by introducing a supervisory controller, it will force the states of the system inside the constraint region that is designed in advance. Therefore, the stability of the closed-loop PID control system is also guaranteed. Finally, the proposed scheme is applied to control a well-known vander pol as an illustrative example. From the simulation results, it can be obviously seen that a satisfactory control performance can be achieved by using the proposed method.

\section{References}

[1] H. Khalil, Nonlinear Systems, Prentice Hall, ISBN-13: 978-0130673893

[2] Wei-Der Chang, Jun-Juh Yan," Adaptive robust PID controller design based on a sliding mode for uncertain chaotic systems",Chaos, Solitons and Fractals 26 (2005) 167-175

[3] Hackl K, Yang CY, Cheng AHD. Stability, bifurcation and chaos of non-linear structures with control-I. Autonomous case. Int J

Non-Linear Mech 1993;28:441. 
[4] Cheng AHD, Yang CY, Hackl K, Chajes MJ. Stability, bifurcation and chaos of non-linear structures with control-II.

Nonautonomous case. Int J Non-Linear Mech 1993;28:549.

[5] Tchoukuegno R, Woafo P. Dynamics and active control of motion of a particle in a / 6 potential with a parametric forcing. Physica D

2002;167:86.

[6] Tchoukuegno R, Nana Nbendjo BR, Woafo P. Linear feedback and parametric controls of vibration and chaotic escape in a $/ 6$

potential. Int J Non-Linear Mech 2003;38:531.

[7] Nana Nbendjo BR, Tchoukuegno R, Woafo P. Active control with delay of vibration and chaos in a double-well-Duffing oscillator.

Chaos Soliton Fract 2003;18:345.

[8] Yamapi R, Nana Nbendjo BR, Enjieu Nkadji HG. Dynamics and active control of a motion of a driven multi-lmit-cycle Van der Pol oscillator. Int J Bifurcation Chaos, in press.

[9] Astrom KJ, Hagglind T. Automatic tuning of simple regulators with specifications on phase and amplitude margins. Automatica 1984;20:645-51 .

[10] Astrom KJ, Hagglind T. Industrial adaptive controllers based on frequency response techniques. Automatica 1991;27:599-609.

[11] Leva, Sung SW. PID autotuning algorithm based on relay feedback. IEE Proceedings - D 1993;140:328-37.

[12] Lee J, Sung SW. Comparison of two identification methods for PID controller tuning. AIChE J 1993;39:695-7.

[13] Wang QG, Zou B, Lee TH, Bi Q. Auto-tuning of multivariable PID controller from decentralized relay feedback. Automatica 1997;33:319-30 .

[14] Tsay DL, Chung HY, Lee CJ. The adaptive control of nonlinear systems using the Sugeno-Type of fuzzy logic. IEEE Trans Fuzzy

[15] Park YM, Choi MS, Lee KY. An optimal tracking neuro-controller for nonlinear dynamic systems. IEEE Trans Neural Netw 1996;7:1099-110 .

[16] Wang LX. Adaptive fuzzy systems and control: design and stability analysis. New Jersey: Prentice-Hall; 1994.

[17] Wang LX. A course in fuzzy systems and control. New Jersey: Prentice-Hall; 1997 .

[18] Chen CT, Peng ST. Intelligent process control using neural fuzzy techniques. J Process Contr 1999;16:493-503 .

[19] Mayosky MA, Cancelo GIE. Direct adaptive control of wind energy conversion systems using Gaussian networks. IEEE Trans Neural Netw 1999;10:898-905 .

[20] Chang WD, Hwang RC, Hsieh JG. Stable direct adaptive neural controller of nonlinear systems based on single auto-tuning neuron. Neurocomputing 2002;48:541-54 .

[21] Lin FJ, Hwang WJ, Wai RJ. A supervisory fuzzy neural network control system for tracking periodic inputs. IEEE Trans Fuzzy Syst 1999;7:41-52. 